\title{
Digestive System Neuroendocrine Tumor G1
}

National Cancer Institute

\section{Source}

National Cancer Institute. Digestive System Neuroendocrine Tumor G1. NCI Thesaurus. Code C7709.

A usually small, slow-growing, well differentiated neuroendocrine tumor arising from the digestive system. It is composed of islands of rounded, oxyphilic, or spindle-shaped cells of medium size, with small vesicular nuclei. The mitotic count is less than 2 per $10 \mathrm{HPF}$ and/or the Ki67 index is equal to or less than 2 percent. The tumor can occur anywhere in the digestive system; approximately $90 \%$ arise in the appendix. 